NASA-TM-111917

\title{
MOTION OF THE HELIOSPHERIC TERMINATION SHOCK AT HIGH HELIOGRAPHIC LATITUDE
}

\author{
Aaron Barnes \\ Theoretical Studies Branch, NASA Ames Research Center, Moffett Field. California. USA
}

\begin{abstract}
We expect the mean distance of the heliospheric termination shock to be greater (smaller) at polar latitudes than at equatorial latitudes, depending on whether the mean dynamic pressure of the solar wind is greater or smaller at high latitudes. The heliospheric termination shock is expected to move in response to variation in upstream solar wind conditions, so that at any particular instant the termination shock will resemble a distorted asymmetric balloon with some parts moving inward and others moving outward. If the shock is a gasdynamic or magnetohydrodynamic shock the results of the analysis depend only very weakly on the nature of the upstream disturbance; typical speeds of the disturbed shock are -100 to $200 \mathrm{~km} / \mathrm{s}$. In the absence of a significant latitude gradient of the typical magnitude of solar wind disturbances typical motions of the disturbed shock at polar latitudes would be about twice as fast, due to the higher speed of the high-latitude wind. If the dynamics of the termination shock are dominated by acceleration of the anomalous component of the cosmic rays, the motion of the shock in response to a given disturbance is substantially slower than in the gasdynamic case. Conceivably, particle acceleration might be a less important effect at higher latitudes, and we envision the possibility of a termination shock that is dominated by particle acceleration at lower latitudes and is an MHD shock at high latitudes. In this event high latitude solar wind disturbances would produce substantially larger inward and outward motions of the shock in the polar regions.
\end{abstract}

\section{Morphology of the Heliospheric Termination Shock}

It is widely accepted that the solar wind flow changes from supersonic to subsonic through a termination shock whose location, roughly speaking, is where the solar wind dynamic pressure is balanced by pressure due to the very local interstellar medium (ISM). A number of physical processes can produce asymmetries in the termination shock. For example, motion of the local interstellar medium toward the heliosphere will tend to compress the heliosphere, including the termination shock, so that the distance to the termination shock will show a distinct fore-aft asymmetry with respect to the direction of the interstellar flow; the magnitude of this asymmetry will be substantial if the flow of the local interstellar medium is supersonic relative to the heliosphere. Asymmetry in the termination shock can originate internally in the solar wind as well as externally in the interstellar medium. It may well be the case that in the dynamic pressure at a given distance is substantially higher (or lower) at polar latitudes than at equatorial latitudes, especially in the years around the solar activity minimum, resulting in a prolate (oblate) figure for the steady-state shock.

Space Science Reviews 72: 233-236.

(1) 1995 Kluwer Academic Publishers. Printed in the Netherlands. 


\section{Motion of the Termination Shock}

In reality the configuration of the termination shock will be much more complicated than the above picture suggests, because the dynamic pressure of the solar wind undergoes large variations on the time scale of days and weeks. Hence the termination shock is unlikely ever to be in equilibrium, and will move inward and outward in response to solar wind variations (e.g., Barnes, 1993; Belcher et al., 1993; Grzedzielski and Lazarus, 1993; Suess, 1993; Whang and Burlaga, 1993). For the most part the solar wind disturbances will be confined to limited ranges of heliographic latitude, so that at a given moment some portions of the termination shock will move outward while others are simultaneously moving inward.

The local response of the termination shock to various upstream disturbances has been analyzed for a pure gasdynamic shock (Barnes, 1993, Naidu and Barnes, 1994a) and a magnetohydrodynamic shock (Naidu and Barnes, 1994b). The results are qualitatively similar in all these analyses, so it is sufficient to review the simplest case, for which the initial upstream disturbance is a gasdynamic contact discontinuity. This disturbance may be parameterized by the density ratio $\rho_{1} / \rho_{\mathrm{u}}$, where the subscript u refers to the solar wind initially just upstream of the termination shock, and the subscript 1 refers to the solar wind upstream of the disturbance. Representative results are given in the first three columns of Table I (adapted from Barnes, 1994). Here $v_{1}$ is the speed of the upstream solar wind, $V_{1}$ is the speed of the termination shock after interaction with the disturbance, and $V_{2}$ is the speed of the downstream propagating signal (a sound wave or weak shock) that carries information of changed upstream conditions into the far downstream medium. Note that $\mathrm{V}_{1}$ is positive (outward propagation) for a density enhancement $\left(\rho_{1} / \rho_{u}>0\right)$, and negative for a density decrease, as one would expect intuitively.

Density disturbances of a factor of order 10 are not unusual in the outer solar wind, so that for $v_{1} \sim 400 \mathrm{~km} / \mathrm{s}$, as is typical of the outer solar wind at equatorial latitudes, one would expect the termination shock to move inward and outward at -100 to $\sim 200$
$\mathrm{~km} / \mathrm{s}$. Thus inward and outward excursions of several AU could be typical. Clearly the first encounter of an outer heliospheric spacecraft with the termination shock would be due to the shock's inward motion past the spacecraft, as opposed to the spacecraft's passing a stationary shock.

Although the trajectory of ULYSSES is limited to the inner heliosphere, highlatitude data from this spacecraft will be relevant to the motion of the termination shock at polar latitudes. Intermediate-latitude ULYSSES observations strongly suggest that at the present epoch the high-latitude solar wind is roughly twice as fast as the equatorial wind (Gosling et al., 1993). Therefore a given enhancement (or decrease) of dynamic pressure would produce an excursion in the termination shock moving roughly twice as fast as the excursion produced by the same variation at equatorial latitudes. Hence it is possible that the excursions of the polar termination shock are systematically larger than the equatorial shock. This conclusion should hold if typical 
TABLE I.

Response to Upstream Disturbance $(\gamma=5 / 3)$

\begin{tabular}{|c|c|c|c|c|c|c|}
\hline & \multicolumn{2}{|c|}{$\lambda=0$} & \multicolumn{2}{|c|}{$\lambda=0.5$} & \multicolumn{2}{|c|}{$\lambda=0.9$} \\
\hline$\rho_{1} / \rho_{u}$ & $v_{1} / v_{1}$ & $\mathrm{~V}_{\text {sig }} / \mathrm{v}_{\mathrm{l}}$ & $\mathrm{v}_{1} / \mathrm{v}_{1}$ & $\mathrm{~V}_{\text {sig }} / \mathrm{v}_{1}$ & $v_{1} / v_{1}$ & $\mathrm{~V}_{\text {sig }} / \mathrm{v}_{1}$ \\
\hline 0 & -2.24 & 0.809 & -1.36 & 0.513 & -0.558 & 0.203 \\
\hline 0.1 & -0.553 & 0.809 & -0.383 & 0.513 & -0.182 & 0.203 \\
\hline .25 & -0.334 & 0.809 & -0.237 & 0.513 & -0.116 & 0.203 \\
\hline 0.5 & -0.166 & 0.809 & -0.121 & 0.513 & -0.061 & 0.203 \\
\hline 1 & 0 & 0.809 & 0 & 0.513 & 0 & 0.203 \\
\hline 2 & 0.160 & 0.895 & 0.123 & 0.592 & 0.066 & 0.251 \\
\hline 4 & 0.309 & 0.985 & 0.245 & 0.684 & 0.137 & 0.313 \\
\hline 10 & 0.485 & 1.10 & 0.401 & 0.816 & 0.242 & 0.419 \\
\hline$\infty$ & 1 & 1.5 & 1 & 1.42 & 1 & 1.35 \\
\hline
\end{tabular}

variations in dynamic pressure at a given heliocentric distance are comparable at polar and equatorial latitudes. On the other hand, it is quite plausible that the flow is substantially smoother at high latitudes, implying smaller variations of dynamic pressure, and mitigating the high-latitude excursions of the termination shock. This issue should be settled by high-latitude ULYSSES plasma observations, at least for epochs not near the solar activity maximum.

\section{Possible Role of Anomalous Cosmic Rays}

So far we have assumed that the termination shock may be modeled as a gasdynamic or magnetohydrodynamic shock. However, it is possible that the heliospheric termination shock is quite different. It is thought that the anomalous component cosmic rays originates as interstellar neutral atoms that penetrate the heliosphere, become ionized and then are accelerated to energies on the order of $100 \mathrm{MeV} /$ nucleon (Fisk et al., 1974), probably at the termination shock itself (e.g., see the reviews of Jokipii (1990) and McKibben (1990)). Extrapolations of outer heliospheric cosmic-ray observations suggest that the energy density of the anomalous component could be comparable to the solar wind energy density within less than 100 astronomical units (AU) from the Sun (Jokipii and Kota, 1990; McKibben, 1990). If so, acceleration of the anomalous component is an essential element in the dynamics of the shock itself.

Barnes (1994) has modeled such a termination shock as a gasdynamic shock modified by inclusion of a sink (representing particle acceleration) in the energy jump conditions. The equilibrium position of this shock is slightly farther from the Sun than in the pure gasdynamic case. The most striking modification, however, is in the response of the shock to upstream disturbances (Table I). The shock is parameterized by the energysink strength $\lambda$ (=rate of energy loss by particle acceleration/rate of flow of energy into 
the shock); $\lambda=0$ is the pure gasdynamic case. It is apparent from the table that for a given upstream disturbance, diversion of energy into particle acceleration tends to reduce the velocity of the disturbed termination shock.

So the termination shock may have its dynamics dominated by acceleration of the anomalous component, or it may be very like a pure gasdynamic shock; for convenience let us label the two kinds of shock as AC and GD, respectively. An interesting speculation is that some portions of the shock may be of the AC variety, others of the GD variety. In fact, according to the models of Donohue and Zank (1993), a shock that is initially $\mathrm{AC}$ may convert to GD and vice versa.

If the distance of the termination shock is of order $100 \mathrm{AU}$, the spiral angle of the heliospheric magnetic field is fairly near 90 degrees even at fairly high latitudes, so that the termination shock on average would be expected to be quasi-perpendicular at essentially all latitudes. On the other hand, if the solar wind is much faster at polar latitudes, the shock transition involves more energy per particle at high latitudes. In addition, heliospheric magnetic fields may deviate considerably from the Archimedean spiral model (Jokipii and Kota, 1989). Such variations might or might not be sufficient to effect the transition between AC and GD, but if the transition can occur it is conceivable that there is a systematic equator-pole transition from one kind of shock to the other. Then, for example, if polar shock tends to be GD and the equatorial shock $\mathrm{AC}$, the polar shock would move back and forth with much larger amplitude than the equatorial shock.

\section{References}

Barnes: A.: 1993. J. Geophys. Res. 98, 15,137.

Barnes. A.: 1994. J. Geophys. Res. 99, 6553.

Belcher, J.W., A.J. Lazarus. R.L. McNutt, Jr., and G.S. Gordon. Jr.: 1993. J. Geophys. Res. 98, 15.177 .

Donohue, D.J., and G.P. Zank: 1993, J. Geophys. Res. 98, 19,005

Fisk. L.F.. B. Koslovsky, and R. Ramaty: 1974, Astrophys. J., Pt. 2 190, L35.

Grzedzielski and Lazarus: J. Geophys. Res. 98, 5551.

Gosling, J.T.: 1994. EOS Trans. Am. Geophys. Un. 74 Supp. , 479.

Jokipii, J.R.: 1990, The Anomalous Component of Cosmic Rays'. in S. Grzedzielski and

D.E. Page (eds.), Physics of the Outer Heliosphere, Pergamon. New York. pp. 169-178.

Jokipii. J.R., and J. Kota: 1989. Geophys. Res. Lett. 16, 1.

Jokipii, J.R., and J. Kota: 1990, Proceedings of the 2/st International Cosmic Ray

Conference 6, 198.

McKibben, R.B.: 1990. 'Cosmic rays in the local interstellar medium', in S. Grzedzielski and

D.E. Page (eds.), Physics of the Outer Heliosphere, Pergamon, New York, pp. 107-118.

Naidu, K., and A. Barnes: 1994a, 'J. Geophys. Res., in press .

Naidu, K., and A. Barnes: 1994b, submitted to J. Geophys. Res.

Suess, S.T.: 1993, J. Geophys. Res. 98, 15,147.

Whang, Y.C., and L.F. Burlaga: 1993, J. Geophys. Res. 98, 15.221. 